\title{
I I Das carmen saeculare des Horaz
}

Die Zeilen I47-I 49 der Inschrift erwähnen die Aufführung des carmen saeculare nach den Opfern des letzten Tages für Apollo und Diana, sie nennen sogar den Autor: carmen composuit Q. Hor[at]ius Flaccus. Den beteiligten je 27 Knaben und Mädchen waren die Voraussetzungen der Aufführung schon vorher durch ein Edikt der Quindecimviri mitgeteilt worden. Sie waren sicher schon einige Zeit vor der Säkularfeier mit dem Einstudieren des Liedes und dem Trainieren des Chores unter Anleitung des Dichters beschäftigt. ${ }^{\mathrm{I}}$ Auch in diesem Fall ist die Quellenlage der augusteischen Säkularspiele beispiellos, weil das carmen saeculare des Horaz im Gesamtwerk des Dichters erhalten ist. Außerdem ist ein Gedicht des Horaz erhalten, in dem er Apollo als Lehrer des Gesanges preist und den Chor der Knaben und Mädchen aus vornehmem Hause ermahnt, seine Aufgabe so zu erfüllen, wie der Ritus es verlangt. ${ }^{2}$ Auch Sueton erwähnt das carmen saeculare in seiner Horazvita. ${ }^{3}$ Diese ausgezeichnete Quellenlage erlaubt, das carmen saeculare im Kontext der gesamten Feier zu interpretieren und seine rituelle Funktion zu bestimmen.

Der Inhalt des carmen saeculare mit seinen neunzehn Strophen ist wegen seiner anfänglichen Unstrukturiertheit nicht einfach wiederzugeben: In den ersten zwei Strophen werden Apollo und Diana gebeten, den Chor zu unterstützen, das Lied zur vorgegebenen Zeit, so wie es die Verse der Sibylle mahnten, zu singen. Ausdrücklich wird in der zweiten Strophe darauf hingewiesen, dass sich das carmen an alle Götter richtet, quibus septem placuere colles. ${ }^{4}$ Apollo und Diana, denen kurz vorher auf dem Palatin das Opfer von je 27 Opferkuchen dargebracht worden ist, werden hier als Patrone heiliger Chöre angerufen. Diese Rolle kommt in besonderem Maße Apollo zu. Ob Apollo in einer Identifikation mit Sol auch die dritte Strophe gewidmet ist, bleibt vorläufig fraglich. In dieser Strophe wird alme Sol gepriesen, der jeden Tag aufs Neue auftaucht und nichts Gewaltigeres auf Erden sieht als die urbs Roma.

I Auf ein vorangegangenes Edikt verweist der Ausdruck in Z. I47 quibus denuntiatum erat. Ein entsprechendes Edikt hat vielleicht in Z. 73-75 vorgelegen. Der praeco-Ausruf geht in Z. $20 \mathrm{f}$. auf den Vortrag des carmen saeculare ein. Vgl. Übersicht S. $38 \mathrm{ff}$. Hor. carm. 4, 6 belegt die Vorbereitung des Chors, ob unter Anleitung von Horaz selbst, ist umstritten, vgl. Anm. 43 dieses Kapitels.

2 Hor. carm. 4, 6, $35 \mathrm{ff} .:$... Lesbium servate pedem meique pollicis ictum, rite Latonae puerum canentes, rite crescentem face Noctilucam ...

3 Suet. vita Hor. p. 46, 2 ff.: scripta quidem eius usque adeo probavit mansuraque perpetua opinatus est, ut non modo saeculare carmen componendum $\langle e i\rangle$ iniunxerit, sed et Vindelicam victoriam Tiberii Drusique privignorum suorum eumque coegerit propter boc tribus carminum libris ex longo intervallo quartum addere.

4 Hor. carm. saec. 7 . 
Erst nach diesen drei Einleitungsstrophen geht das carmen saeculare auf den besonderen Anlass der Säkularspiele ein und erwähnt die Göttinnen, denen die Opfer auf dem Tarentum galten: Ilithyia wird um leichte Geburten zum richtigen Termin gebeten und darum, die Gebärenden zu schützen. Es fällt auf, dass Horaz den Namen von Ilithyia variiert und sie auch Lucina oder Genitalis nennt. 5 Der Grund ist in der Unbekanntheit der drei Ilithyien in Rom zu suchen, die als ursprünglich griechische Geburtsgöttinnen nicht bekannt waren. Diese Funktion lag in Rom eher bei Iuno Lucina oder auch Diana Lucina, an die mit diesen Epitheta erinnert wird. ${ }^{6}$ Der Beiname Genitalis ist zwar für keine andere Göttin überliefert, wird aber aufgrund seiner Etymologie für alle verständlich gewesen sein, um den Ilithyien ein Profil zu geben. ${ }^{7}$ Die folgenden beiden Strophen nehmen noch einmal auf Ilithyia als göttliche Helferin zu einer gesunden Nachkommenschaft Bezug, und insofern werden sie auch als Schutzherrinnen der politischen Beschlüsse des Senats gesehen, die die Förderung des Nachwuchses beabsichtigten. In der fünften Strophe erwähnt Horaz ganz untypisch für einen Hymnus die augusteischen Ehegesetze als Voraussetzung für Nachkommenschaft. ${ }^{8}$ Diese Nachkommenschaft wiederum garantiert, dass auch künftig in I I० Jahren eine Säkularfeier mit Spielen am Tage und in der Nacht gefeiert werden kann (Strophe 6).

Die beiden folgenden Strophen reden die beiden anderen Göttinnen der nächtlichen Opfer auf dem Tarentum an: Die siebente Strophe nennt die Parzen, die in

5 Scheid (2016b) 39-42 erklärt die Wahl der griechischen Namen für die im Tarentum geehrten Göttinnen (Moiren, Ilithyien und Terra Mater = Gaia anstelle der bekannteren Tellus) der Säkularspiele mit einem Konzept, das die augusteische Wiedereinführung der Säkularspiele verfolgte. Dieses von dem Gelehrten Ateius Capito entwickelte Konzept gab den Säkularspielen ein dem hellenistischen Zeitgeist entsprechendes Gesicht. »Manifestament, dans la restauration des Jeux séculaires, le côté grec comptait plus que la réanimation d'une vieille tradition, mais peut-être considerée comme trop banale.« Horaz habe im carmen saeculare mit einer erneuten Transkription der griechischen Götternamen wieder einen Schritt zurück gemacht und damit auf die Unbestimmtheit der Benennungen hingewiesen, gleichzeitig aber mit den lateinischen Namen für Klarheit im Verständnis gesorgt.

6 Dass dieser Beiname beiden Göttinnen zukam, geht aus zahlreichen Weihinschriften hervor: CIL I² 359-362; 37 I; I 58 I. Daneben bei Ter. Andr. 473; Varro ling. 5, 49 und 5, 74. Vgl. Radke (1978) i i I s, Anm. i 22.

7 Flores (1995) I67 glaubt, dass Horaz mit den beiden zusätzlichen Namen darauf hinweisen will, dass es drei Ilithyien gibt.

8 Hor. carm. saec. 17-20: Diva, producas subolem patrumque prosperes decreta super ingandis feminis prolisque novae feraci lege marita. Diese Anspielung auf die jüngste politische Vergangenheit ist von den meisten Interpreten des carmen saeculare als Unterordnung des Horaz unter die Absichten des Augustus gedeutet und deswegen als Phrase ohne jede poetische Kraft gewertet worden. Stellvertretend für viele Fowler (I910) I48: »... that Augustus wrote out in prose what he wanted put into it (the hymn), and that his laureate did this with consummate skill and concinnitas.... At any rate the inspiration came from Augustus and not from the poet's native genius.« 
der Inschrift mit ihrem griechischen Namen Moirae genannt sind. Ihnen soll allgemein ein günstiges Schicksal in Fortsetzung einer glücklichen Vergangenheit angelegen sein. Tellus, die im inschriftlichen commentarium Terra Mater heißt, soll um Fruchtbarkeit auf den Feldern und in den Ställen besorgt sein, deren Wohlergehen auch von den Umweltbedingungen abhängt, die Iuppiter schafft.

Die neunte Strophe kommt wieder auf Apollo und Diana zurück, die schon am Anfang des Gedichtes als Schutzpatrone des Chores erwähnt waren. Apollo soll den Knaben zuhören, Diana den Mädchen. Diese Strophe erhält eine interessante Anknüpfung an die in den Strophen vier bis acht angerufenen Göttinnen der ludi saeculares: Apollo wird als condito mitis placidusque telo angesprochen, womit auf seine Funktion als heilender Apollo hingewiesen wird, der Krankheiten fernhält und in dieser Funktion auch als Bewahrer einer gesunden Nachkommenschaft gesehen werden kann.9 So erweitert Horaz die Rolle Apollos und bindet ihn in die Grundidee der Säkularspiele ein, die dem Erhalt der Nachkommenschaft galt.

Im Weiteren hält sich Horaz an den Hymnenstil und liefert in den Strophen IO-I 2 die Genealogie der gens Iulia, deren Ursprünge auf den trojanischen Flüchtling Aeneas zurückgehen, der sich und die Seinen rettend das etruskische Gestade erreichte und dort den Grundstein zu etwas legte, was größer wurde als das, was er verlassen hatte.

Schon die antiken Kommentatoren des carmen saeculare waren verunsichert, ob die Anfangszeile Roma si vestrum est opus an Apollo und Diana anknüpft oder ob damit wieder wie in Z. 7 alle Götter Roms gemeint seien. ${ }^{10}$ Je nachdem, welche Rolle ein Interpret der Bedeutung Apollos an den Säkularspielen als neuem Staatsgott einräumte, wurde hier auf Apollo und Diana oder alle anderen Götter geschlossen. ${ }^{11}$ In Verbindung mit den folgenden Strophen und der Ankündigung von Z. 7 halte ich es für unwahrscheinlich, dass hier nur Apollo gemeint ist. ${ }^{12} \mathrm{Die}$ Gesamtinterpretation des Liedes wird dies bestätigen. Die Götter werden in Strophe I 2 angerufen, dass sie der Jugend moralische Grundsätze, den Alten Ruhe und der ganzen gens des Romulus, womit die römische Gesellschaft gemeint ist, Wohl-

9 Hor. carm. saec. 33; Wissowa (I912) 294 hält die heilende Funktion für den Grund, warum der Apollokult nach Rom gebracht worden ist. In Rom wurde Apollo in der Frühzeit stets als Heilgott verehrt und erst später in dieser Funktion von Aesculapius konkurrenziert.

Io Porph. Hor. carm. saec. 37: Quare Romam opus Dianae et Apollinis dicat, nondum video; im Weiteren vermutet Porphyrio, dass Horaz auf Vergil zurückgreift, der Aeneas aufgrund eines apollinischen Orakels aus Troja fliehen lässt. In demselben Sinn äußert sich Acro Hor. carm. saec. 37.

I I Pighi (I94I) 2 I I geht davon aus, dass wie in Z. 45 f. und 49 alle Götter gemeint seien, Radke (I978) I I04 glaubt, dass Apollo hier von Horaz als der eigentlich Verantwortliche für die Ankunft der Trojaner in Latium gesehen wird.

I 2 Auch in der Abschlussstrophe des carmen saeculare wird die Hoffnung ausgesprochen, dass Iuppiter und alle Götter das carmen gehört haben, das heißt, dass während des gesamten Gedichts alle Götter als Adressaten des carmen gesehen werden. 
stand, Nachkommenschaft und Ruhm geben. ${ }^{13}$ Die Genealogie gipfelt in dem letzten Spross dieser gens, der als Abkömmling der Venus und des Anchises die Götter mit weißen Rindern ehrt, was jeder Teilnehmer der beiden Opfer für Iuppiter am ersten Tag der Säkularfeier selbst sehen konnte. Daneben aber hat dieser Abkömmling noch eine andere Seite: Er ist als siegreicher Krieger mild, wenn er einmal den Feind unterworfen hat. Diese Strophe mit einer nur indirekten Erwähnung des Augustus leitet zu den außenpolitischen Erfolgen der jüngsten Zeit über, von denen Horaz in der folgenden Strophe spricht: Unterworfen sind Meder, Skythen und Inder, womit auf die Rückgabe der unter Crassus eingebüßten Feldzeichen durch die Parther angespielt wird, die drei Jahre vor den Säkularspielen stattgefunden hatte und als einer der großen außenpolitischen Erfolge dieser Zeit gewertet wurde.

Die nächsten Strophen (I5-19) geben einen Ausblick auf die Geschicke Roms angesichts der Entwicklung unter der trojanischen gens Iulia: Die alten Werte fides, pax, honor, pudor und virtus gelten wieder, Wohlstand kehrt ein. Strophe I6 lenkt wieder auf Apollo, der als wahrsagender und heilbringender Gott mit seiner Kunst gesunderhaltend wirkt. Wenn er die Anstrengungen der Römer zu seiner Verehrung auf dem Palatin sieht, wird er der Verlängerung der Zeit um ein weiteres, noch erfolgreicheres lustrum zustimmen (Strophe 17). ${ }^{14}$ In der gleichen Weise nennt die folgende Strophe Diana, die die Bitten der Quindecimviri und die Gesänge der Kinder erhören wird.

Die letzte Strophe (19) schließt das carmen saeculare mit der Hoffnung ab, dass Iuppiter und alle Götter dieses Lied gehört haben, das ein Chor des Phoebus und der Diana zum Lob der Götter gesungen hat. ${ }^{\text {Is }}$

Das carmen saeculare ist sofort nach Auftauchen der Inschrift zu den augusteischen ludi saeculares einer erneuten Interpretation unterzogen worden. Als Erster hat Mommsen es als Prozessionslied interpretiert, das ausgehend vom $\mathrm{Pa}$ latin auf dem Weg zum Kapitol gesungen wurde. ${ }^{16}$ Mommsen unterstellt Horaz, den Sinn der ludi saeculares nicht verstanden zu haben, weil er die an der Feier geehrten Götter, wie sie in der kurz zuvor gefundenen Inschrift aufgeführt sind, in

I 3 Hor. carm. saec. 45-48: Di, probos mores docili iuventae, di, senectuti placidae quietem, Romulae genti date remque prolemque et decus omne.

I4 In Vers 65 gehe ich von der Textkonstitution Schillings aus (1970) 262-265: Si Palatinas videt aequos aras.

Is Auch diese Strophe enthält die schon in der zehnten Strophe erschienene Zweideutigkeit: Singt ein Chor des Phoebus und der Diana zum Lob dieser beiden oder zum Lob aller Götter? In Erinnerung an Z. 7 ist wohl von Letzterem auszugehen, man sollte aber im Auge behalten, dass Horaz sich mehrfach im carmen saeculare nicht eindeutig ausdrückt, sodass seine Formulierung mehrere Deutungen zulässt.

I6 Mommsen (189I) 256f. (602): Mommsen teilt das Gedicht in drei Abschnitte, deren ersten und dritten er dem Palatin zuordnete, den zweiten dem Kapitol. Um Zwischenstrophen $\mathrm{zu}$ erklären, hält er eine Prozession für möglich, in der der Chor singend und tanzend vom Palatin zum Kapitol über die via sacra zog. 
seinem Lied nicht in gleicher Weise berücksichtigt habe. Damit formulierte er als erster ein Verständnis des carmen saeculare als eine Wiederholung der Riten in gesungenen Versen. ${ }^{17}$ Mommsens Sicht hat sofort viel Widerstand erregt, der einerseits die These des Prozessionsliedes ablehnte, aber auch ein Misslingen des carmen saeculare bestritt. ${ }^{18}$ Allerdings wurde der Ansatz Mommsens nicht grundsätzlich infrage gestellt, nämlich dass ein Kultlied den Ritus einer Feier zu besingen und auf diese Weise noch einmal vorzuführen habe.

Ohne hier eine Wertung über die Qualität des Gedichtes vornehmen zu wollen, soll im Folgenden untersucht werden, was wir über die Aufführungspraxis dieses Gedichts wissen und welche Rolle das Lied an der Säkularfeier spielte. Es geht darum, zu bestimmen, wozu das carmen aufgeführt wurde, ob es eine rituelle Aufgabe zu erfüllen hatte, und wenn, worin diese bestanden hat. Literarische Aspekte des Verständnisses werden hier zugunsten einer inhaltlich-funktionalen Analyse bewusst ausgeklammert, obwohl natürlich gerade die Frage der Gliederung des Gedichts immer wieder von großem Interesse war. ${ }^{19}$ Es scheint mir wichtiger, das carmen saeculare im Hinblick auf die Aussage und Anlage der augusteischen ludi saeculares zu untersuchen, wie wir sie aus den Quellen kennen. Der Fund der Inschrift hat in gewisser Weise an das carmen saeculare die Erwartung herangetragen, dass in ihm das inschriftlich fixierte Ritual noch einmal in Versform erscheinen müsse. Offensichtlich führte diese Erwartung zu keiner Klärung der Funktion des carmen saeculare und damit seines Verständnisses. Als erster Schritt zur Klärung ist es deshalb wichtig, darauf aufmerksam zu machen, was im carmen saeculare gleich und was anders dargestellt ist, als es uns in der Inschrift und den anderen Quellen begegnet. Zunächst die Gemeinsamkeiten:

I. Das carmen saeculare erwähnt das sibyllinische Orakel als Grund für die Einrichtung der ludi (Vers 5). Auch das Orakel spricht von der Notwendigkeit lateinisch gesungener Päane durch Knaben und Mädchen (Zos. 2, 6, ı8-20). Bei

I7 In diesem Sinne meint Rahn (1970) 467, dass der Fund der Inschrift dem Verständnis des carmen saeculare eher geschadet habe.

I 8 Vahlen (1892), Fowler (I910), Fraenkel (1957) 367 ff. Die Diskussion, die auf Mommsens erste Interpretation des carmen saeculare nach dem Fund der Inschrift folgte, gibt Gagé (1972) I 4-i 8 wieder.

I9 Kiessling-Heinze (I9I7) 47If. gehen davon aus, dass das Gedicht in $3 \times 3+3 \times 3$ Strophen + I Abschlussstrophe gegliedert ist; Rahn (1970) 467-479 glaubt an eine Rahmenkonstruktion, während Radke (1978) I I02 annimmt, dass die ersten drei Strophen Apollo und Diana gewidmet sind, die nächsten fünf Strophen den Göttinnen der Opfer auf dem Tarentum, dann wieder drei Strophen für Apollo und Diana, vier Strophen für Iuppiter und Iuno, erneut drei Strophen für Apollo und Diana und eine letzte Abschlussstrophe. Alle diese Einteilungen sind wahrscheinlich aus einer gewissen Ratlosigkeit heraus entstanden, die das Gedicht hinterlässt, wenn man ausgehend von den in den Strophen genannten Göttern eine Linie des Verständnisses sucht. Keine der vorgeschlagenen Gliederungen erscheint mir einleuchtend. 
der Schilderung des Festablaufs beschreibt Zosimus den Chor näher: Am dritten Tage stimmen im Heiligtum des Apollo auf dem Palatin dreimal neun Knaben vornehmer Herkunft und die gleiche Zahl Mädchen, beide Gruppen »ringsumblüht « - das heißt, ihre beiden Eltern sind noch am Leben - griechische und lateinische Hymnen und Päane an, wodurch die den Römern untertänigen Städte in ihrem Wohlstand erhalten bleiben. ${ }^{20}$

2. Das carmen saeculare bestätigt den Abstand von i Io Jahren zwischen zwei Säkularfeiern (Vers 2r).

3. Es nennt das Kollegium der Quindecimviri als das Priesterkollegium, das mit der Durchführung der ludi saeculares betraut ist (Vers 70).

4. Es erwähnt die Dauer der Spiele von drei Nächten und drei Tagen (Verse 23 f.).

In den folgenden Punkten weicht das carmen saeculare jedoch von den anderen Quellen ab:

I. Das carmen saeculare erwähnt die Göttinnen, die in den Nächten auf dem Tarentum Opfer erhalten haben, im Vergleich mit der Inschrift unter anderen Namen und nicht in der Reihenfolge, in der ihnen geopfert wurde (Verse I3-32). Es nennt ihre Funktion, die in der Sicherung einer gesunden Nachkommenschaft, eines günstigen Schicksals des römischen Volkes und der Fruchtbarkeit allgemein liegt.

2. Die kapitolinischen Götter, denen an den ersten beiden Tagen der Säkularspiele geopfert wurde, werden im carmen saeculare nicht namentlich erwähnt. Vers 49 enthält eine Anspielung auf das Opfer eines Ochsen für Iuppiter durch Augustus am ersten Tag und Vers 73 nennt Iuppiter neben allen anderen Göttern als Empfänger des carmen saeculare.

20 Zosimus (2, 5, 5) überliefert, dass der Chor auch in griechischer Sprache gesungen

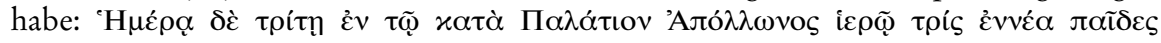

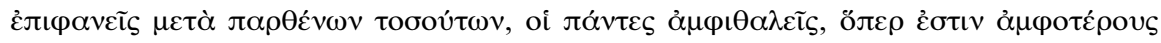

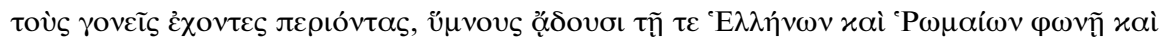

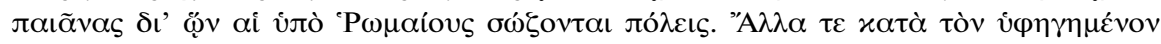

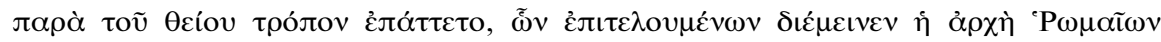
$\dot{\alpha} \lambda \omega ́ \beta \eta \tau$ Tos (Am dritten Tag singen im Heiligtum des Apollo dreimal neun Knaben und ebensoviele Mädchen vornehmer Herkunft in griechischer und lateinischer Sprache Hymnen und Päane; beide Chöre sind »ringsumblüht«, das heißt, ihre beiden Eltern sind noch am Leben. Dadurch bleiben die unter römischer Herrschaft stehenden Städte erhalten. Auch andere Zeremonien wurden auf eine Weise durchgeführt, wie die Gottheit sie vorschreibt. Solange sie ausgeführt wurden, blieb die Herrschaft der Römer unversehrt). Aus dem commentarium der augusteischen und severischen ludi saeculares geht dies nicht hervor. Es ist aber durchaus denkbar, dass neben dem carmen saeculare auch Lieder griechischer Sprache vorgetragen wurden, da die Angaben bei Zosimus meistens ausführlicher sind als die commentaria und sich in den Übereinstimmungen als äußerst präzise erweisen. 
3. Die palatinischen Götter Apollo und Diana werden in den drei Eingangsstrophen angesprochen und während des ganzen Gedichts immer wieder erwähnt. Während der Säkularspiele galten ihnen nur die Opfer des dritten Tages.

In den charakteristischen Grundlagen der ludi saeculares stimmt Horaz mit der restlichen Überlieferung also überein: Der I Io-jährige Abstand von einer Feier zur nächsten, die Quindecimviri als verantwortliches Kollegium, die vorangegangene Befragung des sibyllinischen Orakels und die Dauer von drei Tagen und Nächten.

Die wichtigste Abweichung vom inschriftlich überlieferten Ritual liegt in der Vernachlässigung der kapitolinischen Götter, die niemals direkt angesprochen noch namentlich erwähnt werden. Dagegen werden die auf dem Tarentum geehrten Göttinnen in fünf Strophen erwähnt, wovon allein drei Strophen Ilithyia gelten. ${ }^{21}$ Apollo und Diana werden während des ganzen Gedichts immer wieder angerufen. Wenn man nur das inschriftlich überlieferte Ritual als Quelle heranzieht, ergibt sich auch hier eine deutliche Diskrepanz, die zu klären ist. Es ist kaum davon auszugehen, dass Horaz in eigener Entscheidung dem Lied eine andere theologische Ausrichtung gab, sondern eher, dass sein carmen schon vor der Aufführung dem verantwortlichen Kollegium der Quindecimviri bekannt und auch gebilligt war. ${ }^{22}$ Deswegen sollen im Folgenden die Quellen herangezogen werden, die Auskunft über die Aufführungsbedingungen des Liedes geben und die etwas über die inhaltliche Ausrichtung der ludi saeculares aussagen. Auf diese Weise wird es möglich, im carmen saeculare eine Linie zu erkennen, die dieses Ungleichgewicht der Götter im Vergleich mit der Inschrift erklärt und zeigt, dass es dennoch dem Geist der augusteischen Säkularspiele verpflichtet ist.

Das carmen saeculare wurde am dritten und letzten Tag der ludi saeculares nach Abschluss der Opfer für Apollo und Diana zuerst auf dem Palatin vor dem neuen Apollotempel gesungen, anschließend auf dieselbe Weise auf dem Kapitol. ${ }^{23}$ Ausführende waren je 27 Knaben und Mädchen aus vornehmen Famililen. ${ }^{24}$ Außerdem mussten sie patrimi et matrimi sein, das heißt, Vater und Mutter mussten noch am Leben sein, was einer kultischen Qualifikation entsprach. ${ }^{25}$ Über die Art und Weise der Aufführung gibt es kaum Hinweise, die severische Inschrift liefert

2 I Dies wurde durchgehend als Ungenügen des carmen saeculare verstanden. Niemals wurde dieser Tatbestand zum Anlass genommen, über die religösen Vorausetzungen, unter denen Horaz das Lied schrieb, nachzudenken. Diese waren möglicherweise nicht dieselben, wie diejenigen, die erkennbar sind, wenn man nur die Inschrift als Quelle heranzieht.

22 Darauf lässt die Erwähnung des Orakels in Vers 5 schließen, welches Horaz offenbar kannte.

23 Z. I 47 f.; Zos. 2, 5, 5 dagegen nennt nur den Palatin als Aufführungsort.

24 Hor. carm. 4, 6, 3 I f.: virginum primae puerique claris patribus orti; Act. Sev. 234f.;

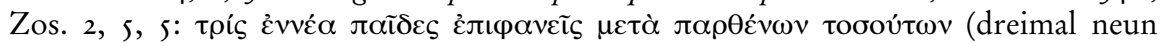
Knaben und ebenso viele Mädchen vornehmer Herkunft).

25 Vgl. den Sachkommentar zu Z. 20, S. 9if. 
zusätzlich einige Angaben über die Kleidung: ${ }^{26}$ Die Knaben des severischen carmen saeculare waren mit der toga praetexta bekleidet und einem Kranz auf dem Kopf, die Mädchen waren mit einer palla ausgestattet und ins Haar geflochtenem Schmuck. Während des Singens hielten sie sich an den Händen und führten Tanzschritte aus. ${ }^{27}$ Diese Bedingungen trafen auch für den Vortrag des carmen saeculare des Horaz zu.

Der Zeitpunkt des ersten Vortrags ist in der augusteischen Inschrift mit sacrificioque perfecto angegeben - also nach Beendigung des Opfers. ${ }^{28}$ Mit sacrificium ist das Opfer von je 27 Opferkuchen für Apollo und Diana gemeint. Nach dem zweiten Vortrag auf dem Kapitol fanden wie nach allen Opfern der vorangegangenen Tage Säkularspiele im Tarentum und sellisternia der Matronen auf dem Kapitol statt. ${ }^{29}$ Die ludi scaenici sind am letzten Tag durch Wagenrennen und Kunstreiter erweitert worden. ${ }^{30}$ Das bedeutet, dass der zweimalige Vortrag des carmen saeculare in das Ritual der Spiele, zwischen Opfer, Spielen und sellisternia, eingebettet war und nicht als eine poetische Zusatzleistung gedacht war. ${ }^{3 \mathrm{I}}$ Dafür spricht vor allem die Vorschrift eines Kultliedes durch das sibyllinische Orakel. ${ }^{32}$ Die Frage muss allerdings gestellt werden, welche Aufgabe es als Kultlied hatte, ob es einen bestimmten Zweck erfüllte oder ob es zwar als Ritual gedacht war, diese Funktion aber nicht erfüllte. ${ }^{33}$

26 Act. Sev. $234 \mathrm{f}$.

27 Darauf verweist Horaz in der Einstudierungsode 4, 6, 35 f., wie er mit dem Daumen den Takt angibt: Lesbium servate pedem meique pollicis ictum. Vgl. den Sachkommentar zu Z. I47-I49, S. I 55, Anm. 392.

28 Z. I47; ebenso Act. Sev. 233 f.: Die severische Inschrift erwähnt allerdings, dass die beiden Augusti Septimius Severus und Caracalla unmittelbar nach dem Apollo-Opfer ins Tarentum gegangen sind und dort im provisorischen Theater aus Holz Säkularspiele angeschaut haben. Anschließend sind sie wieder auf den Palatin zurückgekehrt, wo das carmen saeculare zur Aufführung kam.

29 Z. I 33 f.: die Inschrift erwähnt die sellisternia der Matronen nicht, es ist aber klar, dass solche sellisternia für Iuno und Diana in jeder Nacht und an jedem Tag stattfanden, vgl. den Sachkommentar zu Z. I 5-19, S. 89 ff. Act. Sev. 259 f. erwähnen die sellisternia des dritten Tages nach der Aufführung des carmen saeculare.

30 Z. I 53 f. Das severische commentarium (Act. Sev. 266-272) protokolliert diese abschließenden Spiele nach dem Vortrag des carmen saeculare und den sellisternia der Matronen.

3 I Fraenkel (1957) 380f. hält das carmen vom religiösen Standpunkt aus für überflüssig. Gegen Fraenkel wendet sich Canzik (1996) rosf., der mit Recht betont, dass zwar die Opfer des dritten Tages beendet waren, aber noch nicht die Spiele.

32 Zos. 2, 6, 20-22.

33 Diese Position wird von Feeney (1998) 35 ff. vertreten, der als Hauptaufgabe des carmen saeculare eine Latinisierung der sonst vom griechischen Ritus bestimmten Feier sieht. Er schreibt dem carmen eine begleitende Funktion zu, die zwischen Ritus und Dichtung liegt. Als wichtigsten Beweis sieht Feeney die Tatsache, dass das carmen der augusteischen ludi saeculares nicht in der Inschrift zitiert wird, sondern dass nur seine Aufführung inschriftlich bestätigt wird. Damit anerkennt er eine Mittelstellung 
Das Singen von Kultliedern ist nicht nur aus dem Kult anderer Priesterkollegien bekannt, sondern auch zu bestimmten zweckgebundenen Anlässen. Die Aufführungsbedingungen des carmen saeculare weisen keine Ähnlichkeit mit den durch die commentarii der Arvalbrüder überlieferten Aufführungen des carmen arvale auf, sondern eher mit einigen bei Livius überlieferten carmina, die in Rom aus Anlass einer Bedrohung aufgeführt wurden. ${ }^{34}$ So wurde im Jahre 207 v. Chr. nach mehreren schlechten Vorzeichen wie Steinregen und der Geburt eines Zwitterwesens auf Anweisung etruskischer haruspices und Beschluss der pontifices eine Prozession von dreimal neun Jungfrauen veranstaltet, die durch die Stadt gehend ein Lied sangen, welches Livius Andronicus geschrieben hatte. ${ }^{35}$ Weil diese Vorzeichen sich nach Meinung der haruspices auf die Matronen bezogen, brachten 25 ausgewählte Matronen der Stadt Rom der Iuno auf dem Aventin eine goldene Schale dar, die sie aus ihrer Mitgift finanziert hatten. Anschließend wurden noch einmal Opfer für Iuno angesetzt, die Opfertiere dafür - zwei weiße Färsen wurden prozessionsartig vom Apollotempel vor der porta Carmentalis in die Stadt geführt. Zu dieser Prozession gehörte wieder ein Chor von 27 Jungfrauen, die mit langen Gewändern bekleidet, ein Lied zu Ehren von Iuno sangen. Den Jungfrauen folgten die mit Lorbeer bekränzten Quindecimviri in der toga praetexta. Auf dem Forum formierten sich die virgines zu einem Tanz, den sie mit stampfenden Füßen ausführten, während sie alle durch ein Seil miteinander verbunden waren, an dem sie sich hielten. ${ }^{36}$ Anschließend opferten die Quindecimviri die zwei Opfertiere vor dem Tempel der Iuno Regina auf dem Aventin. Eine ähnliche Prozession fand aufgrund häufiger Missgeburten im Jahre $200 \mathrm{v}$. Chr. statt, allerdings wurde diese Prozession von ebenfalls 27 Jungfrauen von den Quindecimviri nach Befragung der sibyllinischen Bücher angeordnet. ${ }^{37}$

Diese Berichte bei Livius weisen in einigen Aufführungsbedingungen und im Anlass des Vortrags interessante Parallelen zum carmen saeculare auf: Die gleiche

des carmen: Es war durch seine Stellung im Festablauf in den rituellen Zusammenhang integriert, hatte aber einen eher erklärenden und interpretierenden Charakter, der für Rituale nicht zwingend ist.

34 Scheid (1990) 616-623; das carmen arvale war eng mit dem Kultort verbunden und scheint zu jeder Feier im gleichen uralt (scheinenden) Wortlaut gesungen worden zu sein, den die Arvalen aus vorher verteilten Abschriften kannten. Das carmen saeculare dagegen wurde nicht am Ort, der mit dem Kult der Säkularspiele verbunden war, aufgeführt, sondern im Zentrum der Stadt Rom und war speziell aus Anlass der augusteischen Spiele gedichtet worden, wie auch die severischen Säkularspiele ihr eigens für diesen Anlass gedichtetes carmen saeculare hatten. Zu Kultliedern bei besonderen Anlässen: Liv. 27, 37, 4-I 5; 3 I, I 2, 8, abgedruckt bei Pighi (I94 I) I $97 \mathrm{ff.}$

35 Liv. 27, 37, 7: Decrevere item pontifices, ut virgines ter novenae per urbem euntes carmen canerent. Id cum in Iovis Statoris aede discerent conditum ab Livio poeta carmen, tacta de caelo aedis in Aventino Iunonis Reginae.

36 Liv. 27, 37, I4: In foro pompa constitit, et per manus reste data virgines sonum vocis pulsu pedem modulantes incesserunt.

37 Liv. 3 I, I 2, 8. 
Zahl der Singenden geht vermutlich auf alte Regeln des kultischen Tanzes zurück. Wichtig ist, dass ein lebender Dichter das Kultlied für den besonderen Anlass gedichtet hat, der in beiden Fällen in der Gefährdung der Reproduktion lag. ${ }^{8}$ Der Ritus scheint am Ende des dritten Jahrhunderts in Rom neu eingeführt worden zu sein, worauf die erste Anweisung durch etruskische haruspices und die anschließende Anweisung durch die Quindecimviri hinweist. Es scheint damit ein Ritus eingerichtet worden zu sein, mit dem auf einmalige Ereignisse, die in besonderem Maße die Matronen betrafen, reagiert werden konnte. Diese Einbeziehung von Frauen in Riten der römischen Religion ging auf den zunehmenden Einfluss der griechischen Religion in Rom zurück, wo die Teilnahme von Frauen an religiösen Zeremonien eine gängige Praxis war. Wohl aus diesem Grund waren die Quindecimviri zuständig.

Die Aufführung des carmen saeculare, die in einer ähnlichen Form stattfand und das auch von einem lebenden Dichter für den Anlass der ludi saeculares geschrieben wurde, stellt vermutlich eine bewusste Anknüpfung an diese Riten dar, um auf das gemeinsame Ziel hinzuweisen: die Sicherung gesunden Nachwuchses. Die Exegeten der augusteischen ludi saeculares waren sich wohl bewusst, dass hier ein Ritus vorlag, der ursprünglich nicht mit den ludi saeculares verbunden war, weswegen das carmen saeculare nicht im Tarentum aufgeführt wurde. Aber mit diesem Ritus bestand die Möglichkeit, die Aussage der ludi saeculares in die Stadt zu holen und auch am Tage zu wiederholen. Dieser Bezug wurde hergestellt, indem ein Ritual, das sonst nur anlässlich einer akuten Bedrohung durchgeführt wurde, aber dasselbe Ziel verfolgte wie die ludi saeculares, in die Abfolge der Riten der ludi saeculares gestellt wurde. Das carmen saeculare war der einzige Ritus der drei Festage auf dem Kapitol und auf dem Palatin, der überhaupt Aussagen zu der religiösen Ausrichtung der Säkularfeier machte und Elemente dieser Feier zur Sprache brachte. Die Opfer an Iuppiter, Iuno und Apollo/Diana stellten keinen direkten Bezug zur Aussage der Säkularfeier her, sie waren Staatsopfer, wie sie an anderen großen Festen auch stattfanden. Einzig der Vortrag des carmen saeculare nahm die besonderen Gedanken der Säkularfeier auf: Die Wiederholung nach i ı Jahren, die vorangegangene Befragung des sibyllinischen Orakels, das Kollegium der Quindecimviri, die vielleicht auch eine Funktion während des Vortrags des carmen saeculare hatten und die Erwähnung der Göttinnen, denen in den vorangegangenen Nächten auf dem Tarentum geopfert worden war.

Eine Besonderheit des carmen saeculare stellt die Besetzung des Chors dar, der nicht nur aus Jungfrauen bestand, sondern zugleich aus 27 Knaben. Die Frage, was diese Abweichung von den überlieferten Kultliedern zu bedeuten hat, ist in der bisherigen Untersuchung des carmen saeculare nicht verfolgt worden. ${ }^{39}$ Es werden

38 Für das Lied des Jahres 200 v. Chr. ist der Dichter umstritten. Vgl. Pighi (I94I) I98f.

39 Vgl. Schmidt (1985) $48 \mathrm{ff}$;; Schmidt weist darauf hin, dass durch die Beteiligung von Knaben eine neue Aufführungschoreografie wahrscheinlich wurde, wie sie Doppel- 
nicht allein aufführungstechnische Gründe vorliegen, sondern diese Erweiterung des Chors hat einen inhaltlichen Grund: Vielleicht sollte die Beteiligung von 27 Knaben den rein auf Frauen bezogenen Rahmen der Fruchtbarkeit erweitern. Der Fortbestand Roms sollte nicht nur als rein biologische Dimension gesehen werden, sondern durch die Beteiligung der Knaben auch in seiner politischen Dimension wahrgenommen werden. Rom sollte weiter Bestand haben durch genügend Nachkommen und durch seine bewährte militärische Stärke. Diesen Gedanken enthält auch das carmen saeculare, wenn es sagt: Romulae genti date remque prolemque et decus omne ( $47 \mathrm{f}$.)..$^{\circ}$

Somit kann als eine der Funktionen des carmen saeculare die Erweiterung des Kultes vom Tarentum in das Gebiet der Stadt gelten. Dies geschah, indem die traditionelle Form eines bestehenden Kultliedes für die ludi saeculares nutzbar gemacht wurde. Das carmen saeculare ist zwar nicht direkt ein Teil der überlieferten Riten der Säkularfeier, ihm war aber mit Sicherheit eine rituelle Funktion zugedacht, was die Parallelen zu diesen alten Kultliedern zeigen. Die rituelle Funktion erhielt das carmen saeculare, indem an einen bestehenden Ritus, der dem Gedanken der ludi saeculares nahestand, aber in keiner Weise mit dem Kultort der Spiele verbunden war, anknüpfte. Andererseits schafft das carmen saeculare selbst eine solche Anknüpfung, indem es die grundlegenden Riten der ludi saeculares aufnimmt; dazu gehören das sibyllinische Orakel, die Quindecimviri, nächtliche Opfer an die Göttinnen des Tarentum. Die Frage, ob diese Art von ritueller Einbeziehung als solche zu werten sei oder eine künstliche Erweiterung darstelle, scheint mir nicht richtig gestellt zu sein: Von der Anlage her gehörte das carmen zu den Riten, keiner der Teilnehmer der Säkularfeier dürfte daran gezweifelt haben. Es wurde als eine alte Form wahrgenommen, die schon lange existierte. Horaz selbst scheint von der rituellen Funktion seines Gedichts überzeugt gewesen zu sein, wie sich noch zeigen wird. Die Inschrift der severischen ludi saeculares hat das carmen saeculare in die Riten aufgenommen, denn sein Inhalt wird vollständig in der Inschrift wiedergegeben. ${ }^{41}$

chöre ermöglichen. Die einzelnen Strophen den beiden Chorhälften plausibel zuzuteilen, wie Schmidt es versucht, halte ich für schwierig. Klar ist nur, dass beide Chöre getrennt agierten, wie es schon im Orakel gefordert war.

40 Dass mit decus wohl die militärische Stärke gemeint ist, kommt in Strophe I 3 zum Ausdruck, welche die Milde des siegreichen Augustus gegenüber den Besiegten preist.

4I Act. Sev. 235-247. Feeney (1998) 37f. hatte eine solche inschriftliche Fixierung des horazischen carmen saeculare vermisst, um es vollständig den Riten der Säkularfeier zuordnen zu können. Es ist aber nicht die Überlieferungssituation, die ein Element einer Feier zu einem Ritus macht, sondern die selbstverständliche Ausführung. Dass der Vortrag des carmen saeculare diese Stellung erreicht hatte, geht daraus hervor, dass die severischen Spiele es so selbstverständlich aufgenommen haben. Leider sind wir für die dazwischen liegenden Spiele unter Domitian nicht informiert, aber es ist anzunehmen, dass auch an dieser Feier in irgendeiner Form der Ritus eines carmen saeculare vollzogen wurde. 
Wie diese Einbindung des Kultliedes in die ludi saeculares durch das carmen saeculare aussah, lässt sich aber nicht nur durch die Übernahme der Formen der Aufführung feststellen, sondern auch durch den Text des carmen saeculare selbst, der in vielem Bezüge zu dem Kult der Säkularspiele enthält. Die augusteischen Spiele verehrten mit den nächtlichen Opfern im Tarentum Moiren, Ilithyia und Terra Mater, beziehungsweise die im carmen erwähnten Göttinnen Ilithyia, Parzen und Tellus. Nur das carmen erwähnt diese Göttinnen mit der Funktion, die sie an den Säkularspielen hatten. Während die Rituale auf dem Tarentum die Göttinnen mit einem stets gleichen Gebet ansprachen, in welchem ganz allgemein um Fortbestand, Überlegenheit und Wohlergehen des römischen Volkes gebetet wurde, macht das carmen Unterschiede und geht auf die je unterschiedliche Funktion der Göttinnen ein. Auch die Inschrift macht keinerlei Angaben über die Funktion der Göttinnen. Wie wir gesehen haben, war die Göttin Ilithyia nicht in Rom bekannt, was besonders aus den Versen I3-17 des carmen saeculare hervorgeht. Somit erläutern die Angaben im carmen saeculare, was von den verschiedenen Opfern und Gebeten der letzten drei Nächte zu erwarten ist.

Ilithyia wird an erster Stelle genannt, weil ihre Aufgabe die vordringlichste der Säkularspiele ist. Sie erhält ein ausgeprägteres Profil als die Parzen und Tellus, weil die Erläuterung ihrer Funktion für die Säkularspiele wichtig ist. Auf diese Ausrichtung der Säkularspiele scheint Horaz gleich am Anfang einzugehen und es ist dieser Gedanke, der im Zusammenhang mit den Göttinnen des Tarentum am meisten Raum erhält. ${ }^{2}$

Dass Horaz den Gedanken der Reproduktion als den Grundgedanken der Säkularspiele verstand, geht auch aus der Interpretation der Vorbereitungsode zum carmen saeculare hervor. ${ }^{43}$ In dieser Ode, die an Apollo gerichtet ist, findet sich ein Exkurs über Achill, dessen Verbindung mit der abschließenden kurzen Ermahnung des Chores bisher nicht deutlich genug gesehen worden ist. ${ }^{44}$ Achill wird Apollo als unverständlich grausam gegenübergestellt, obwohl auch Apollo als Rächer seiner von Niobe gekränkten Mutter grausam sein kann. Die noch größere Grausamkeit des Achill wird in ihrer Erbarmungslosigkeit selbst Kindern gegenüber geschildert. Achill hätte sogar die im Mutterleib geschützten Kinder dem Verderben preisgegeben, hätten nicht die Götter eingegriffen, und hätte nicht

42 Hor. carm. saec. I $3-24$.

43 Hor. carm. 4, 6; Fraenkel (1957) 403 ff. hält es zu Recht für ausgeschlossen, dass Horaz selbst das Lied mit dem Chor einstudierte, er hält Vers 35 für eine Metapher, die den Chor zum Beachten des Versmaßes auffordert, so wie er es fixiert hatte. Die Rolle, in

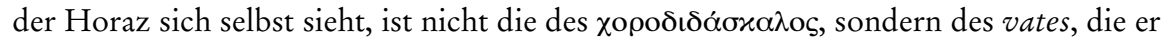
sich in der Sphragis der letzten Strophe selbst gibt: carmen docilis modorum vatis Horatii (carm. 4, 6, 43 f.).

44 Kiessling-Heinze (I9I7) zu carm. 4, 6 Achillexkurs; auch Fraenkel (I957) 400ff. meint, dass die ersten sechs Strophen des Gedichts wenig mit dem Schlussteil zu tun haben und ein entlegenes Thema behandeln. 
Apollo den tödlichen Pfeil des Paris gegen Achill gelenkt.45 Der Grundtenor dieses Vergleichs ist die Unantastbarkeit des noch ungeschützten Lebens der Kinder. Apollo ist zwar grausam, aber gerecht. Daneben aber ist er als doctor argutae fidicen Thaliae der Patron der Dichtkunst, weswegen er von Horaz um Gelingen des carmen saeculare angerufen wird. Horaz weist ausdrücklich darauf hin, dass die von Achill beabsichtigten Opfer noch unfähig waren zu sprechen (carm. 4, 6, I 8: nescios fari pueros). Damit spielt er auf die Funktion der künftigen Choristen an, die für den Vortrag des carmen wichtig sind. Nicht nur das Leben dieser Kinder wurde von Apollo gerettet, sondern auch ihre künftige Funktion sichergestellt, den Göttern einen Hymnus zu singen. Der Vergleich schafft eine inhaltliche Beziehung zum carmen saeculare, indem es auf die Voraussetzungen für das Singen des Hymnus eingeht. Es unterstreicht mit einem Beispiel aus der Mythologie, dass auch im grausamsten kriegerischen Wüten die Unantastbarkeit der Nachkommen respektiert werden muss, weil das deren sicheres Heranwachsen die Erfüllung künftiger religiöser Pflichten gewährleistet. Das Vorbereitungslied zum carmen saeculare nimmt in gewisser Weise dieses Grundthema voraus, stellt es aber unter einen anderen Aspekt, nämlich die Bedeutung der Nachkommen schon als Kinder für die Ausübung der Religion und insbesondere die Bedeutung der künftigen Sänger und Sängerinnen des carmen saeculare.

Diese Zukunftssicherung durch die Nachkommen kommt auch in der letzten der drei Ilithyia gewidmeten Strophen zum Ausdruck: Dass künftig Säkularspiele gefeiert werden können, hängt von dem segensreichen Wirken Ilithyias ab, die nicht nur direkt auf die Geburten einwirken, sondern auch alle Maßnahmen unterstützen soll, die diesem Ziel verpflichtet sind. ${ }^{46}$ Unter diese Maßnahmen nimmt Horaz die Senatsbeschlüsse für die Ehegesetze des Augustus auf, die er hier decreta super ingandis feminis prolisque novae feraci lege marita nennt. ${ }^{47}$ Diese deutliche Anspielung auf die Zeitgeschichte hat Horaz vonseiten der Interpretatoren Unverständnis und den Vorwurf der Liebedienerei gegenüber Augustus eingetragen. ${ }^{4}$ Dass der Name des Augustus ausdrücklich im ganzen Gedicht vermieden wird, erschien den Interpretatoren zusätzlich noch heuchlerisch. ${ }^{49}$

Es ist bekannt, dass Horaz sich Augustus verweigert hat, als dieser ihn bat, den vertrauensvollen Posten seines Privatsekretärs zu erfüllen. ${ }^{\circ}$ Offenbar hat er aber

45 Hor. carm. 4, 6, I8-20: nescius fari pueros Achivis ureret flammis, etiam latentem matris in alvo.

46 Hor. carm. saec. 2 I-24 zu künftigen Spielen, I7-20 zu den Ehegesetzen des Augustus.

47 Es handelt sich um die lex Iulia de maritandis ordinibus und um die lex Iulia de adulteriis coercendis, die wohl beide aus dem Jahre i 8 v. Chr. stammen. Vgl. Kienast (2014) I65ff.

48 Mommsen (I89ib) 357; Fowler (I910) I48f.; Flores (I995) I67.

49 Die Vermeidung der Namensnennung von Augustus wird besonders in Z. 49f. deutlich, wo Augustus clarus Anchisae Venerisque sanguis genannt wird.

so Suet. vita Hor. p. 45 , 7 ff. 
im Fall der Säkularspiele doch eine Aufgabe übernommen und zusätzlich in einer weiteren Ode darüber berichtet. Das lässt vermuten, dass er der Aufgabe, ein carmen zu den ludi saeculares zu schreiben, mit Stolz nachgekommen ist. Horaz hat in dieser Aufgabe eine Erfüllung seiner Berufung zum vates des populus Romanus gesehen..$^{5 \mathrm{I}}$ Er verstand sich als Interpret der Feierlichkeiten des Tarentum, und dazu gehörte, die Ereignisse der jüngsten Geschichte einzubeziehen. Die unmittelbar zuvor erlassenen Ehegesetze, die in der Inschrift ebenfalls erwähnt sind, eigneten sich, die aktuelle politische Entwicklung im Sinne der Säkularfeier $\mathrm{zu}$ interpretieren..$^{52}$ Es ist bekannt, dass sich die Ehegesetze in der senatorischen Schicht und wohl auch bei Horaz als Junggesellen keiner Beliebtheit erfreuten. Die hier gebotene Einbettung der Gesetze in religiöse Vorstellungen könnte als Versuch gewertet werden, die hinter den Gesetzen stehende Verpflichtung, legitime Nachkommen zu zeugen, in einem größeren Zusammenhang zu verstehen und zu akzeptieren. Auch wenn es uns befremdlich scheinen mag, dass Horaz auf diese Weise Gesetze des Augustus akzeptabel machen wollte, müssen wir die Möglichkeit in Rechnung stellen, dass das an den ludi saeculares vertretene Anliegen dem Dichter selbst eingeleuchtet hat. Er erwähnte wohl die Ehegesetze ohne Zwang vonseiten der Quindecimviri oder von Augustus. ${ }^{33}$ Dass er von dem Grundgedanken der ludi saeculares überzeugt war, könnte man bereits aus der vorbereitenden Ode zum carmen saeculare schließen, aber noch mehr aus der Tatsache, dass er diesen Auftrag überhaupt angenommen hat. ${ }^{54}$

Sein Verständnis des Auftrags als eine besondere religiöse Aufgabe stützen die Überlieferungssituation und ein weiteres Zeugnis: Das carmen saeculare ist nicht ins Korpus der anderen carmina eingegliedert, sondern in allen Handschriften gesondert überliefert. Dies lässt auf eine Sonderstellung schließen, die bereits Horaz selbst diesem carmen zuschrieb." Außerdem spricht er in der an Augustus gerichteten Epistel über seine Rolle als Dichter und insbesondere als Dichter von kultischen Gedichten. ${ }^{6}$ Diese Zeilen spielen unmissverständlich auf das drei Jahre

s H Hor. carm. 4, 6, 44.

52 Z. 52-57. Diese Zeilen enthalten den Senatsbeschluss, der denen, die durch die lex de maritandis ordinibus gebunden sind, dennoch die Teilnahme an den ludi saeculares erlaubt.

53 Die von Horaz gewählte Formulierung decreta super ingandis feminis (V. I $8 \mathrm{f}$.) macht deutlich, dass er an dieser Stelle in erster Linie Frauen als Betroffene der Ehegesetze sah. Auch dies zeigt, dass er in den Ehegesetzen eher Maßnahmen zur Geburtenförderung gesehen hat als eine Eheverpflichtung.

54 Auch der antike Kommentar zum carmen saeculare erwähnt als eine Motivation der Säkularfeier die Eindämmung und Abwehr von Seuchen, also ebenso die Sicherung des Fortbestands der Bevölkerung; Schol. Hor. (pseudoacroniana) carm. saec. I: saecularis carminis duplex devotio esse consueverat: aut enim pro sedanda et vertenda pestilentia, aut pro certo et constituto numero annorum.

55 Vgl. Canzik (1996) I07.

56 Hor. epist. 2, I, I32-I 38 . 
vorher aufgeführte carmen saeculare an. ${ }^{57}$ Horaz sieht im Rückblick seine dichterische Funktion in der Verstärkung der Bitten, die durch die dichterische Sprache eine zusätzliche Kraft gewinnen. Dies geschieht durch die direkte Ansprache der Götter: carmine di superi placantur, carmine Manes. ${ }^{8}$ Damit gibt Horaz selbst dem carmen saeculare eine kultische Funktion, die er erreicht, indem ihm Apollo zu einem Ausdruck verhilft, der über das normale Sprechen hinausgeht.

Die besondere Rolle, die neben Apollo auch Diana im carmen saeculare spielt, wurde als eine Folge des unter Augustus geförderten Apollokultes gewertet. 59 Die Hervorhebung des Apollokultes kommt zwar an den Opfern der Säkularspiele selbst zum Ausdruck, wenn neben den Staatsgöttern Iuppiter und Iuno auch Apollo und Diana am dritten und wichtigsten Tag Opfer erhalten, allerdings keine blutigen Opfer, sondern nur Opferkuchen. ${ }^{60}$ Die Rolle von Apollo im carmen saeculare ist nicht aus diesem Grund so überragend. Apollo erhält seine Präsenz im carmen saeculare durch seine besondere Funktion als Schutzherr des Chores und des Dichters, in diese Funktion wird Diana mit einbezogen. Die Eingangsbitte des carmen saeculare formuliert dies deutlich: date, quae precamur tempore sacro, quo Sibyllini monuere versus virgines lectas puerosque castos dis, quibus septem placuere colles, dicere carmen. Die letzte Strophe nimmt diese Bitte wieder auf, wenn der Chor sich als Phoebi chorus et Dianae bezeichnet. Die Vorbereitungsode zum carmen saeculare hebt diese für Horaz wichtige Funktion Apollos am deutlichsten hervor. Sie richtet sich vom ersten Wort an (dive) an Apollo, der gebeten wird, dem Dichter bei seiner wichtigen Aufgabe zu helfen. ${ }^{61}$ Horaz sieht in ihm den Gott, der ihm als Mittler zur Seite steht, damit das Lied, welches an alle Götter gerichtet ist $(7 \mathrm{f}$.$) , von diesen auch vernommen wird \left(73 \mathrm{f}\right.$.). ${ }^{62}$

57 Besonders die Erwähnung des Knaben- und Mädchenchors in Hor. epist. 2, I, I 32 f. weist darauf hin: castis cum pueris ignara puella mariti disceret unde preces, vatem ni Musa dedisset? Aber auch die Wirkung der dichterischen Worte entspricht den an der Säkularfeier ausgesprochenen Wünschen (epist. 2, I, I35-I38): caelestis inplorat aquas docta prece blandus, avertit morbos, metuenda pericula pellit, impetrat et pacem et locupletem frugibus annum.

58 Hor. epist. 2, I, I 38.

59 Radke (1978) i I05 ff.

60 Erst der Fund der Inschrift offenbarte, dass Apollo und Diana mit Opferkuchen geehrt wurden; das Orakel der ludi saeculares, das schon vorher bekannt war, spricht dem Apollo dieselben Opfer wie Iuppiter und Iuno zu - ein Rind (Zos. 2, 6, I6-I8). Diese Abweichung lässt sich auf einen Kompilationsfehler zurückführen, der bei der Redaktion des Orakels für die Säkularspiele durch die Einarbeitung bestehender Verse unterlaufen ist (vgl. S. 242). Feeney (1998) glaubt, dass Horaz im carmen saeculare in Vers 49 vom Text des Orakels ausgeht und sich auch dieser Vers auf Apollo und Diana bezieht. Nach der vorangegangenen Strophe halte ich dies nicht für möglich, weil Horaz in $45 \mathrm{ff}$. ebenso wie in der letzten Strophe alle Götter anspricht, an deren Spitze die kapitolinischen Götter stehen.

6 I Hor. carm. 4, 6, 24-3I.

62 Ebenso Gagé (I972) $23 \mathrm{f}$. 
In Zusammenhang mit der Bedeutung Apollos im carmen saeculare wird immer wieder die dritte Strophe angeführt, in welcher alme Sol angesprochen ist. Schon die antiken Kommentare setzten Sol mit Apollo gleich. Dies, weil das sibyllinische Orakel Phoebus Apollo auch Helios nennt. ${ }^{63}$ Für eine solche Identifikation spricht der Beitrag Fowlers, der auf das fastigium des Apollotempels auf dem Palatin hinweist, welches mit einer Figur des Sol mit einer Quadriga geschmückt war. ${ }^{64}$ Auf diese Giebelfigur könnten diese Verse anspielen. Gegen eine solche Gleichsetzung von Apollo mit Sol spricht sich Galinsky aus. ${ }^{65}$ Er macht geltend, dass besonders das Epitheton alme auf Sol als selbstständigen Gott hinweise. Almus ist hier in der Bedeutung fertilis, uber, fecundus verstanden und reiht Sol als einen der für die Fruchtbarkeit wichtigen Götter in das carmen ein. Da auch nach Meinung von Galinsky proles das Hauptthema des carmen saeculare ist, passt die Erwähnung von Sol im carmen. ${ }^{66}$ Auch wenn wir davon ausgehen müssen, dass Horaz aufgrund seiner Kenntnis des sibyllinischen Orakels von der Identifikation Apollo - Sol wusste, so würde ich die Frage, wer mit alme Sol gemeint ist, offen lassen. Vielleicht hat Horaz selbst absichtlich einen Ausdruck gewählt, der verschiedene Assoziationen zuließ, die sowohl von dem Ort des Vortrags inspiriert sein konnten als auch von den theologischen Kenntnissen des Einzelnen.

Ebenso wie die starke Bewertung der Rolle Apollos an den Säkularspielen hat sich eine Sicht der ludi saeculares als Feier zum Beginn eines Goldenen Zeitalters etabliert. $\mathrm{Da}$ in die Argumentation dafür auch das carmen saeculare einbezogen wurde, soll an dieser Stelle darauf eingegangen werden. Diese Sicht der augusteischen Säkularspiele hat sich bis in die neuere Forschung erhalten, ohne dass wir in den Quellen zu den ludi saeculares und auch im carmen saeculare selbst jemals das Wort 'golden< oder Ähnliches finden. ${ }^{67}$ Barker ist der Herkunft des Begriffs aurea aetas nachgegangen und hat nur zwei Texte gefunden, die dafür verantwortlich sind, dass diese Vorstellung mit den ludi saeculares verbunden wurde: Es handelt sich um die vierte Ekloge Vergill ${ }^{68}$ und die immer wieder zusammen mit den ludi saeculares zitierte Stelle der Aeneis:

63 Zos. 2, 6, i6f.

64 Fowler (I910) I 5 I. Bezeugt ist diese Figur durch die Verse des Properz 3, 28 ff.: Tum medium claro surgebat marmore templum et patria Phoebo carius Ortygia. In quo Solis erat supra fastigia currus.

65 Galinsky (1967) 619-633.

66 Galinsky (I967) 627f.; die Erwähnung von Sol hat nach Galinsky drei Absichten verfolgt: Sie schafft ein Gegengewicht zu den folgenden Muttergottheiten mit einem pater Sol, sie schafft eine Verbindung zu Aeneas, denn der latinische Sol Indiges wurde mit Aeneas Indiges gleichgesetzt, und sie erinnert an die vielfältigen Beziehungen zwischen Latinern und Römern, auf die während der Säkularfeier immer wieder angespielt wird.

67 Barker (I996) 434-446 führt besonders Zanker (1987) I7 I-I77 an.

68 Besonders Verg. ecl. 4, 4-Io; die Entstehungszeit der vierten Ekloge scheint in den vierziger Jahren des letzten vorchristlichen Jahrhunderts mit der Erwartung eines neuen saeculum zusammen zu fallen. Das letzte saeculum hatte I 49 v. Chr. begonnen, 
Hic vir, hic est, tibi quem promitti saepius audis, Augustus Caesar, divi genus, aurea condet saecula qui rursus Latio regnata per arva Saturno quondam, super et Garamantas et Indos proferet imperium; (Aen. 6, 79I-795)

Weder das Goldene Zeitalter noch andere mit Namen von Metallen überlieferte Zeitalter werden im carmen saeculare genannt. Es ist auch nichts über Augustus' eigene Vorstellungen vom Goldenen Zeitalter bekannt. ${ }^{69}$ Barker erläutert, dass der Begriff aurea aetas in augusteischer Zeit einen beweglichen Diskurs darstellte, der aus vielen verschiedenen Quellen gespeist wurde, aber auch in vielerlei Gestalt auftauchte. ${ }^{70}$ Mit der Vorstellung eines neuen Goldenen Zeitalters war immer eine Wiederkehr moralischer Werte verbunden, wie sie auch von Horaz im carmen saeculare beschrieben wird. Es ist aber doch wichtig, diese Erneuerung von fides, pax, honos, pudor und virtus in dem Kontext zu lesen, den Horaz bietet: Er erwähnt die moralische Erneuerung nicht als Folge eines goldenen saeculum, sondern als Folge der Errungenschaften des römischen Volkes. Diese sind die Eroberungen fremder Völker, die als Besiegte geschont werden, und der Einsatz der Menschen (45-56). Barker erscheint gerade das Fehlen jeglicher Anspielungen auf ein Goldenes Zeitalter merkwürdig. Seiner Meinung nach grenzt sich Horaz dadurch von der negativen Konnotation des Begriffs >Gold< ab. In einem parallel zur aurea aetas verlaufenden Diskurs gegen Luxus wurde das einfache Leben gepriesen und Gold als Quelle der Vernachlässigung innerer Werte gesehen. ${ }^{71}$ Horaz spricht ausdrücklich nicht von aurea copia, sondern von beata copia, womit er auf die tatsächlich gesprochenen Gebete der Säkularfeier anspielt und schlicht konventionelle Fruchtbarkeit meint, für welche die Göttinnen der nächtlichen Opfer stehen. Die Goldlosigkeit der copia im carmen saeculare steht als Symbol für die mora-

somit war die Erwartung eines neuen saeculum nach etwa hundert Jahren groß. Siehe Pighi I4f. - mit den Scholien zu Vergil, die das Auftauchen des Kometen erwähnen.

69 Galinsky (198I) I 93 weist auf das Fehlen entsprechender Quellen hin, führt aber aus, dass die Verknüpfung von Goldenem Zeitalter mit augusteischer Zeit wahrscheinlich von Augustus unterstützt wurde. Galinsky führt in seinem Aufsatz die von Barker auf das carmen saeculare übertragene Fragestellung für die Behandlung des Goldenen Zeitalters bei Ovid durch und kommt wie Barker zu der interessanten Beobachtung, dass Ovid gerade durch seinen Gebrauch dieses Begriffs in einer für die augusteische Zeit untypischen Weise sich der offiziellen Sicht verschließt.

70 Barker (I 996) 436f. weist darauf hin, dass wir die Komplexität, die der Begriff aurea aetas unter Augustus angenommen hatte, auf zahlreichen Gebieten beobachten können: Friedenspolitik, Landwirtschaft, Glücksvorstellungen etc. Dennoch ist es nicht möglich, Abhängigkeiten und Entwicklungen zu rekonstruieren. Die Idee vom Goldenen Zeitalter existierte, sie erhielt durch ihre Übertragung auf alle Gebiete des politischen und gesellschaftlichen Lebens jedoch gleichzeitig eine Pervertierung.

7I Ovid, Ars Amatoria 2, 275 f.: Carmina laudantur, sed munera magna petuntur: Dummodo sit dives, barbarus ipse placet. Aurea sunt vere nunc saecula. Plurimus auro venit honos. Auro conciliatur amor. 
lische Güte des neuen saeculum. Das Goldene Zeitalter wird absichtlich nicht proklamiert, weil durch den Begriff sgolden< die moralische Tugend des saeculum zweifelhaft wird. Darin liegt eine Realitätsbezogenheit des carmen saeculare, die $\mathrm{ihm}$ in den meisten bisherigen Interpretationen als Einverständnis mit der herrschenden Ideologie vorgeworfen wurde. Man kann diese Realitätsbezogenheit aber auch als eine Absage an übertriebene Erwartungen und eine Rückbesinnung auf die Grundlagen einer Gesellschaft verstehen.

Das carmen saeculare war ein Ritus der Säkularspiele und in die Abfolge der Riten eingegliedert. Es gehörte nicht zu den ursprünglich mit Säkularfeiern verbundenen Riten, seine Aufführung stellt eine der augusteischen Erneuerungsmaßnahmen der Feier dar. Wie es im Gesamtablauf der Feier wahrgenommen wurde, ist nicht mehr zu rekonstruieren, der Ursprung eines solchen Kultliedes dürfte nur den theologisch Gebildeten bekannt gewesen sein. Durch die Anknüpfung an bereits bestehende Formen solcher Kultlieder, wurde der Eindruck verstärkt, dass es sich um eine althergebrachte Angelegenheit handelte, zumal das Singen von Kultliedern zu mehreren Festen gehörte. Andererseits hat Horaz allein durch die Wahl des Versmaßes, aber auch durch die Anspielungen auf die Zeitgeschichte den Rahmen eines archaischen Kultliedes gesprengt. Hier sollte nicht etwas Uraltes vorgetäuscht, sondern eine neue Aussage in einer neuen Form gefunden werden, die auf gewisse überlieferte Parallelen zurückgreifen konnte. Insofern ist das carmen saeculare ein gutes Beispiel dafür, wie die augusteische Erneuerung der Religion ausgesehen hat. Weil Horaz selbst das carmen saeculare als ein Kultlied aufgefasst hat, eignet es sich nicht wie andere Gedichte, Aussagen über die politische Haltung von Horaz aus ihm zu schließen. Er hat seinen Auftrag hier nicht in der Äußerung eigener Gedanken gesehen, sondern in der Formulierung von Bitten, die er kraft des dichterischen Wortes verstärkte. Offenbar war er über die augusteische Rekonstruktion der Feier vorher gut unterrichtet worden, sodass er sich mit der Idee der Säkularfeier auseinandersetzen konnte. Es war den Rekonstrukteuren der Feier gelungen, auch Horaz in den Dienst eines so allgemeinen Gedankens wie das Fortbestehen Roms durch eine gesicherte Nachkommenschaft zu stellen. Hinter dieser unbestreitbar positiven Idee gerieten die Mittel der Politik zur Durchsetzung dieser Idee in den Hintergrund. 